\title{
Predictive models of control strategies involved in containing indoor airborne infections
}

\begin{abstract}
Recently developed control measure modeling approaches for containing airborne infections, including engineering controls with respiratory protection and public health interventions, are readily amenable to an integrated-scale analysis. Here we show that such models can be derived from an integrated-scale analysis generated from three different types of functional relationship: Wells-Riley mathematical model, competing-risks model, and Von Foerster equation, both of the key epidemiological determinants involved and of the functional connections between them. We examine mathematically the impact of engineering control measures such as enhanced air exchange and air filtration rates with personal masking combined with public health interventions such as vaccination, isolation, and contact tracing in containing the spread of indoor airborne infections including influenza, chickenpox, measles, and severe acute respiratory syndrome (SARS). If enhanced engineering controls could reduce the basic reproductive number $\left(R_{0}\right)$ below 1.60 for chickenpox and 3 for measles, our simulations show that in such a prepared response with public health interventions would have a high probability of containing the indoor airborne infections. Combinations of engineering control measures and public health interventions could moderately contain influenza strains with an $R_{0}$ as high as 4 . Our analysis indicates that effective isolation of symptomatic patients with low-efficacy contact tracing is sufficient to control a SARS outbreak. We suggest that a valuable added dimension to public health inventions could be provided by systematically quantifying transmissibility and proportion of asymptomatic infection of indoor airborne infection.
\end{abstract}

\section{S-C. Chen, C-F. Chang, C-M. Liao}

Ecotoxicological Modeling Center, Department of Bioenvironmental Systems Engineering, National

Taiwan University, Taipei, Taiwan

Key words: Indoor Airborne Infection; Basic Reproductive Number; Asymptomatic Infection; Mathematical Models; Indoor Air Quality.

Chung-Min Liao

Ecotoxicological Modeling Center

Department of Bioenvironmental Systems Engineering National Taiwan University

Taipei 10617

Taiwan

Tel.: +886 223634512

Fax: +886 223626433

e-mail: cmliao@ntu.edu.tw

Received for review 14 September 2005. Accepted for publication 9 March 2006.

(c) Indoor Air (2006)

\section{Practical Implications}

We have developed a flexible mathematical model that can help determine the best intervention strategies for containing indoor airborne infections. The approach presented here is scalable and can be extended to include additional control efficacies. If a newly emergent airborne infection should appear, the model could be quickly calibrated to data and intervention options at the early stage of the outbreak. Data could be provided from the field to estimate value of $R_{0}$, the serial interval between cases, the distributions of the latent, incubation, and infectious periods, case fatality rates, and secondary spread within important mixing groups. The combination of enhanced engineering control measures and assigned effective public health interventions would have a high probability for containing airborne infection.

\section{Introduction}

Airborne transmission is known to be a route of infection for diseases. About 15 million (>25\%) of 57 million annual deaths worldwide are estimated to be related directly to infectious diseases. Of those infectious disease-related deaths 4 million annual deaths $(7 \%)$ occur as a consequence of viral respiratory infections (WHO, 2004). Worldwide, for example, severe influenza infections develop in 3-5 million people annually, and 250,000-500,000 deaths occur (WHO, 2003b). Control of newly emerging airborne infections such as severe acute respiratory syndrome
(SARS), potential bioterror agents such as smallpox and pandemic influenza (Fauci et al., 2005; Morens et al., 2004) have generated an even more acute sense of the need to analyze airborne infection transmission. The transmission route for SARS demonstrated that the higher transmission probability was probably caused by environmental air movements and has an important relationship with ventilation airflow ( $\mathrm{Li}$ et al., 2005a,b; Wong et al., 2004; Yu et al., 2004). The threat that a highly transmissible, highly virulent respiratory disease agent can present, such as SARSassociated coronavirus, and for which there is no effective developing vaccine or antimicrobial drug, 


\section{Chen et al.}

requires decision makers to give nonvaccine airborne infection much greater consideration in community settings than has been traditionally given.

Reducing indoor airborne infection risk had been an important factor in the developments of layout and ventilation schemes of public health settings. In light of the series of studies that attempted to identify the major sources and routes of indoor airborne infectious particle transmission, engineers have to recognize that, for both air and direct contacts, adequate statistical evidence has been available to support the importance of providing a bacteria-free microenvironment (Beggs, 2003; Chow and Yang, 2004; Lee et al., 2005). Because the occurrence of respiratory disease is thought to be related to the amount of contagion in the air, engineering controls such as air dilution and ventilation, filtration, dust suppression, and air sterilization, which attempt to decrease the concentrations of microorganisms in the air, theoretically constitute effective nonvaccine infectious acute respiratory disease interventions (Lee et al., 2005).

How do we quantify the possible risks of an epidemic of indoor airborne infection, and how can we evaluate different control measures that might impact on any given indoor airborne infection during its emergence and early-stage growth? Simple mathematical analysis can provide some insights. We use mathematical models to give structure and clarity to the analysis of the key epidemiological determinants of the transmission dynamics of major indoor airborne infections and evaluate what interventions worked best in different settings. We are trying to present analyses of data on key parameters and distributions and discuss the processes of data capture, analysis and the impact of public health measures against indoor airborne infection.

In this paper, we propose an integrated-level mathematical model generated from three different types of functional relationship: Wells-Riley mathematical model, competing-risks model, and Von Foerster equation, both of the key epidemiological determinants involved and of the functional connections between them. The proposed model links approximate analytical methods and a relatively parsimonious stochastic individual-based simulation model embedding estimates of transmission efficiency and the details concerning the typical course of infection to explore both what works best and in what combination, and the degree to which a specific intervention must be applied in order to evaluate how different control measures might impact on any given airborne infection during its emergence and early-stage growth.

Competing-risks theory (Brookmeyer et al., 2003, 2004, 2005) is used to account for the impact of different enhanced measure efficacies from both engineering controls and respiratory protection on the airborne infection risk. The selected optimal control measures include environmental controls by ventilation and air filtration, as well as respiratory protection.
Inclusion of competing risks in the model recognize that an individual may face substantial benefits in risk reduction of airborne infection from many different control measures including technological controls at the source (by surgical mask and treatment booths), environmental controls (by ventilation, air filtration and ultraviolet germicidal irradiation), and receptor controls (by respiratory protection via respirators) (Barnhart et al., 1997; Chow and Yang, 2004; Myatt et al., 2004; Nardell et al., 1991; Nazaroff et al., 1998; Rudnick and Milton, 2003).

Fraser et al. (2004) have derived a Von Foerster equation-based criteria for outbreak control in that they adopted two key properties of transmission of basic reproductive number $\left(R_{0}\right)$ and the proportion of asymptomatic infections that arise prior to the onset of symptoms $(\theta)$ to analyze the general properties of directly transmitted agents that determine the likely success of certain public health measures for containing early-stage outbreaks. They indicated that $R_{0}$ and $\theta$ are both strong predictors for successfully describing the impact of simple public health control measures against the infectious diseases. By estimating $R_{0}$ and $\theta$ for SARS, human immunodeficiency virus-1, smallpox, and influenza A, they concluded that SARS and smallpox are easier to control using the simple public health measures of isolation and contact tracing and quarantining.

Fraser et al. (2004) and Anderson et al. (2004) have also given some insights that by some combination of certain efficacious control measures, we could decide whether such public health measures would work effectively if applied with a given efficacy to control the spread of specific pathogens with defined biological and epidemiological properties. We employ the Wells-Riley mathematical model of airborne infection (Fennelly et al., 2004; Rudnick and Milton, 2003) to estimate the exposure concentrations in indoor environments where cases of inhalation airborne infection occurred based on reported epidemiological data and epidemic curves and $R_{0}$ and its variability in a shared indoor airspace.

The objective of this paper was to establish a general quantitative framework that can help predict whether simple control measures including engineering controls and public health interventions or some specific combinations can be successful in containing epidemic growth of indoor airborne infections if applied efficaciously at an early-stage outbreak. To focus the analysis, simulations are based on the highly disseminated epidemic of indoor airborne infections in Taiwan including SARS, influenza, chickenpox, and measles as a model. We interpret these results in terms of the generic performance of future control measure purposes in which appropriate interventions of highly effective strategies for implementing them are prepared before the start of the epidemic. 


\section{Materials and methods}

Epidemiology data of influenza, chickenpox, measles, and SARS

In the present study, we focus quantitatively on reanalysis of epidemiological data of influenza, chickenpox, measles, and SARS that are highly disseminated epidemics of indoor airborne infections in Taiwan. Influenza caused 20-40 million deaths in the 1918 pandemic, and continues to kill thousands each year (Mills et al., 2004); moreover, the viral strains and influenza season varying from country to country have made influenza a still uncontrollable disease. Chickenpox has a high transmission rate between children in school settings. Measles is a generally mild disease, yet its very high incidence among healthy children gives rise to considerable morbidity. Infectious acute respiratory diseases have impact on human health. SARS is a recently described illness of humans with a high case fatality rate (Donnelly et al., 2003) that has spread widely since November 2002. Taiwan had the third highest number of cumulative SARS cases and SARS deaths in the world (WHO, 2003a). Moreover, more than $90 \%$ of SARS cases in Taiwan have been linked to hospital settings (WHO, 2003c).

With the detailed epidemiological data from reported cases regarding influenza, chickenpox, measles, and SARS associated with epidemic curves (numbers of cases by date/week/month of symptom onset), we estimated the quantum generation rate for selected indoor airborne infections in the absence of interventions and control efforts based on infected probability estimated from Wells-Riley equation. Infectivity data of influenza and chickenpox were adopted from the Center for Disease Control of Taiwan based on a weekly basis number from January 2003 to April 2005. A daily basis infectivity data for measles were adopted from Riley et al. (1978) in a suburban elementary school setting. Because there is relatively little empirical data of distribution of measles case in Taiwan, accordingly, we must rely on whatever empirical data are available. Epidemiology of SARS reported by Taipei Municipal Ho-Ping Hospital from April 24 to May 8, 2003 were used in the present study.

Highly infectious risk frequently happens as a result of person-to-person transmission in indoor environments; hence, we used a real ventilation scenario to simulate the control efficacy. The indoor environment regarding engineering control information of influenza and chickenpox was adopted from the Construction and Planning Agency, Ministry of Interior, China (http://www.cpami.gov.tw/pwi/br/br_26-1.php). For SARS and measles, engineering control data were adopted from the Taipei Municipal Ho-Ping Hospital and a typical elementary school setting, respectively.

Because of limited knowledge, both from observations and theoretical understanding of transmission in a ventilated airspace, we need to characterize uncer- tainty and variability. We used a Monte Carlo simulation to quantify our uncertainty concerning infection probability and quantum generation rate. We used the Kolmogorov-Smirnov (KS) statistics to optimize the goodness-of-fit of distributions. We employed Crystal Ball software (Version 2000.2; Decisioneering, Inc., Denver, CO, USA) to analyze data and to estimate distribution parameters. For this study, 10,000 iterations are sufficient to ensure stability of results.

Parameter estimates for $R_{0}$ and $\theta$ by Wells-Riley mathematical equation

The key epidemiological variable that characterizes the transmission potential of a disease is the basic reproductive number $\left(R_{0}\right)$, which is defined as the average number of successful secondary infection cases generated by a typical primary infected case in an entirely susceptible population. $R_{0}>1$ implies that the epidemic is spreading within a population and that incidence is increasing, whereas $R_{0}<1$ means that the disease is dying out. An average $R_{0}$ of 1 means the disease is endemic equilibrium within the population. $R_{0}$ essentially determines the rate of spread of an epidemic and how intensive a policy will need to be to control the epidemic.

Fraser et al. (2004) give the other key variable determining the controllable level of infectious disease, $\theta$, the proportion of asymptomatic infection, which determines the potential for symptom-based public health control measures to reduce the number of infections. Fraser et al. (2004) pointed out that the choice of parameter $\theta$ has the key advantage that at the start of an outbreak it can readily be estimated by using contact tracing as it is the proportion of infections occurring with an asymptomatic infector.

We modify the Wells-Riley mathematical equation proposed by Rudnick and Milton (2003) to estimate the transmission potential of four selected indoor airborne infections. The modified Wells-Riley mathematical equation expresses transmission potential of infection as a function of the fraction of inhaled air that has been exhaled previously by someone in the building (i.e., rebreathed fraction) using $\mathrm{CO}_{2}$ concentration as a marker for exhaled-breath exposure.

Rudnick and Milton (2003) proposed a Wells-Riley mathematical equation as:

$$
P=\frac{D}{S}=1-\exp \left\{-\frac{I q p t}{Q}\left[1-\exp \left(-\frac{Q t}{V}\right)\right]\right\},
$$

where $P$ is the probability of infection for susceptible population, $D$ is the number of disease cases, $S$ is the number of susceptible population, $I$ is the number of infectors, $q$ is the quantum generation rate by an infected person (quanta/h), $p$ is the breathing rate per person $\left(\mathrm{m}^{3} / \mathrm{h}\right), t$ is the total exposure time (h), $Q$ is the outdoor air supply rate $\left(\mathrm{m}^{3} / \mathrm{h}\right)$, and $V$ is the volume of the ventilated space $\left(\mathrm{m}^{3}\right)$. 


\section{Chen et al.}

The outdoor air supply rate $(Q)$ can be expressed as functions of the fraction of indoor that is exhaledbreath $(f)$, people in the ventilation airspace $(n)$, and the breathing rate per person $(p)$, as $Q=n p / f$ based on the total $\mathrm{CO}_{2}$ level in the indoor air contributed from human origin and outdoor air supply and person-toperson transmission of infectious diseases through the recirculation air in the ventilation airspace. When we consider $I=1$ and $S=n-1$ and incorporate $Q=n p /$ $f$ into Equation $1, R_{0}$ for an airborne infection in a building environment can be derived from Equation 1 and expressed as,

$$
\begin{aligned}
R_{0}= & (n-1)\left\{1-\exp \left\{\frac{-q f t}{n}\right.\right. \\
& {\left.\left.\left[1-\frac{V f}{n p t}\left(1-\exp \left(-\frac{n p t}{V f}\right)\right)\right]\right\}\right\} . }
\end{aligned}
$$

Parameter estimates for $\theta$ of four selected indoor airborne infections including SARS, measles, chickenpox, and influenza are determined by the specific biological characteristics of the etiological agents. By the definition of $\theta=$ (incubation period - latent period)/infectious period, we can calculate the distribution of $\theta$ using the Monte Carlo simulation technique to quantify the uncertainty concerning $\theta$ attributable to large variance of incubation period, latent period, and infectious period for a specific indoor airborne infection. The period from the point of infection to the appearance of symptoms of disease is termed the incubation period, whereas the period from the point of infection to the beginning of the state of infectiousness is termed the latent periods. The incubation, latent period, and infectious periods are respectively $1-4,1-3$, and 4 8 days for influenza, 8-13, 6-9, and 6-7 days for measles, and 13-17, 8-12, and 10-11 days for chickenpox (Anderson and May, 1991).

\section{Competing-risks model}

In this study, we adopted the concept of competing-risks model (Brookmeyer et al., 2003, 2004, 2005) to account for the impact of different enhanced measures from engineering controls and respiratory protection on the indoor airborne infection risk. The competing-risks model is a probabilistic model by which the dynamics of the interplay among different enhanced engineering control measure strategies can be described. Implicitly, the basic assumption of the probabilistic calculations on competing-risks model was based on an underlying Poisson model in that the different engineering control measures act independently of each other.

We link the competing-risks model and Wells-Riley mathematical equation to estimate the reduction of potential infectious force of $R_{0}$. Based on the competing-risks model, the optimal $R_{0}$ can be obtained by incorporating the effectiveness of engineering control measures including recirculation filter, air change rate, and the respiratory protection into Equation 2, as

$$
\begin{aligned}
R_{0}= & (n-1)\left\{1-\exp \left[-\left(\frac{\operatorname{Iqtp}\left(1-\eta_{\mathrm{s}}\right)}{Q+Q_{\mathrm{r}} \eta_{\mathrm{r}}}\right)\right.\right. \\
& \left.\left.\left(1-\exp \left(-\left(\mathrm{ACH}+\mathrm{ACH}_{\mathrm{r}} \eta_{\mathrm{r}}\right) t\right)\right)\right]\right\},
\end{aligned}
$$

where $Q_{\mathrm{r}}$ is the airflow rate through a recirculation filter $\left(\mathrm{m}^{3} / \mathrm{h}\right), \eta_{\mathrm{s}}$ is the efficiency of a respiratory protection device used by a susceptible person (dimensionless), $\eta_{\mathrm{r}}$ is the single-pass removal efficiency for infectious droplet nuclei passing through the recirculation filter (dimensionless), $\mathrm{ACH}$ is the air change rate $\left(\mathrm{h}^{-1}\right), \mathrm{ACH}_{\mathrm{r}}$ is the air change rate through a recirculation filter $\left(\mathrm{h}^{-1}\right)$.

\section{$R_{0}-\theta$ critical control line by Von Foerster equation-based model}

We adopted the Von Foerster equation-based control model (Fraser et al., 2004) to analyze the impact of the combinations of non-engineering control measures including isolation, contact tracing, and vaccination. The Von Foerster equations are usually used to determine the disease spread dynamics of the current number of people $Y$ at time $t$ who were infected time $\tau$ ago as $Y(t, \tau)$ (Murray, 2001). Based on the Von Foerster equation-based model, Fraser et al. (2004) show how the two key parameters $R_{0}$ and $\theta$ can be used to predict whether control policies involving isolation and contact tracing will lead to outbreak containment. Accordingly, following the parameter estimates for $R_{0}$ and $\theta$, we can construct the $R_{0}-\theta$ critical control line from the control measure efficacy and estimate $R_{0}$ value from modified Wells-Riley equation of four selected indoor airborne infections of influenza, chickenpox, measles, and SARS.

Based on the $R_{0}-\theta$ critical control line, for each scenario, if a given infectious agent is below the $R_{0}-\theta$ curve, the outbreak is always controlled eventually, whereas above the curve, additional control measures would be required to control spread. The critical control line dividing outbreak control from epidemic growth can be determined by the following equation (Fraser et al., 2004),

$$
\begin{aligned}
& R_{0}\left[\left(1-\varepsilon_{1}\right)\left(1-\varepsilon_{2}\right)+\varepsilon_{1}\left(1-\varepsilon_{2}\right) \theta+\left(1-\varepsilon_{1}\right) \varepsilon_{2} \theta\right. \\
& \left.\quad+\varepsilon_{1} \varepsilon_{2} \frac{\theta}{2-\theta}\right]=1
\end{aligned}
$$

where $\varepsilon_{1}$ and $\varepsilon_{2}$ represent the two different control measure effectiveness assigned for four indoor airborne infections. Equation 4 is originally calculated from following algebraic equation as:

$$
\int_{0}^{\infty} \beta(\tau)\left[1-\varepsilon_{1}+\varepsilon_{1} S(\tau)\right]\left[1-\varepsilon_{2}+\varepsilon_{2} S(\tau)\right] \mathrm{d} \tau=1,
$$


together with an assumption of the exponential infectiousness distribution of $\beta(\tau)=R_{0} \mathrm{e}^{-\tau}$ where $\beta(\tau)$ represents infectiousness at time $\tau$ since they were infected and $S(\tau)$ is the cumulative density function of the incubation period distribution that represents the proportion of people not having symptoms by time $\tau$. Fraser et al. (2004) assume that all susceptible individuals are equally likely to become infected. In addition, make the unrealistic but simplifying assumption that the infectiousness distribution is exponential.

To adopt an appropriate control measure, we have to seek the balance rigorously between the control costs and infectious risks, yet there are many control measure strategies that had been deliberately discussed including the engineering control, public health interventions, and vaccination. However, control measure efficacy, control measure cost, and intervention time by government, as well as quantification of the control measure effectiveness are still an important issue. We divided control measure strategies into three categories of engineering, public health interventions, and specific combination of engineering controls and public health interventions to simulate the variation of $R_{0}-\theta$ curve and to estimate the uncontrollable level.

\section{Results}

Epidemic curves and quantum generation rates

Based on the epidemic curves for cases reported for influenza, measles, chickenpox, and SARS (Figure 1a-d), the probability distribution of quantum generation rates ( $q$, quanta/h) could be calculated from the modified Wells-Riley equation (Equation 1) together with the estimated probability of infection $(P)$ and the adopted input parameters (Table 1). Probabilities of infection for influenza, measles, chickenpox, and SARS shown in Table 1 were estimated based on statistical criteria, comparisons of distribution parameters, and visual interpretation of histograms and resulted in the lognormal (LN) distribution optimizing the KS statistics.
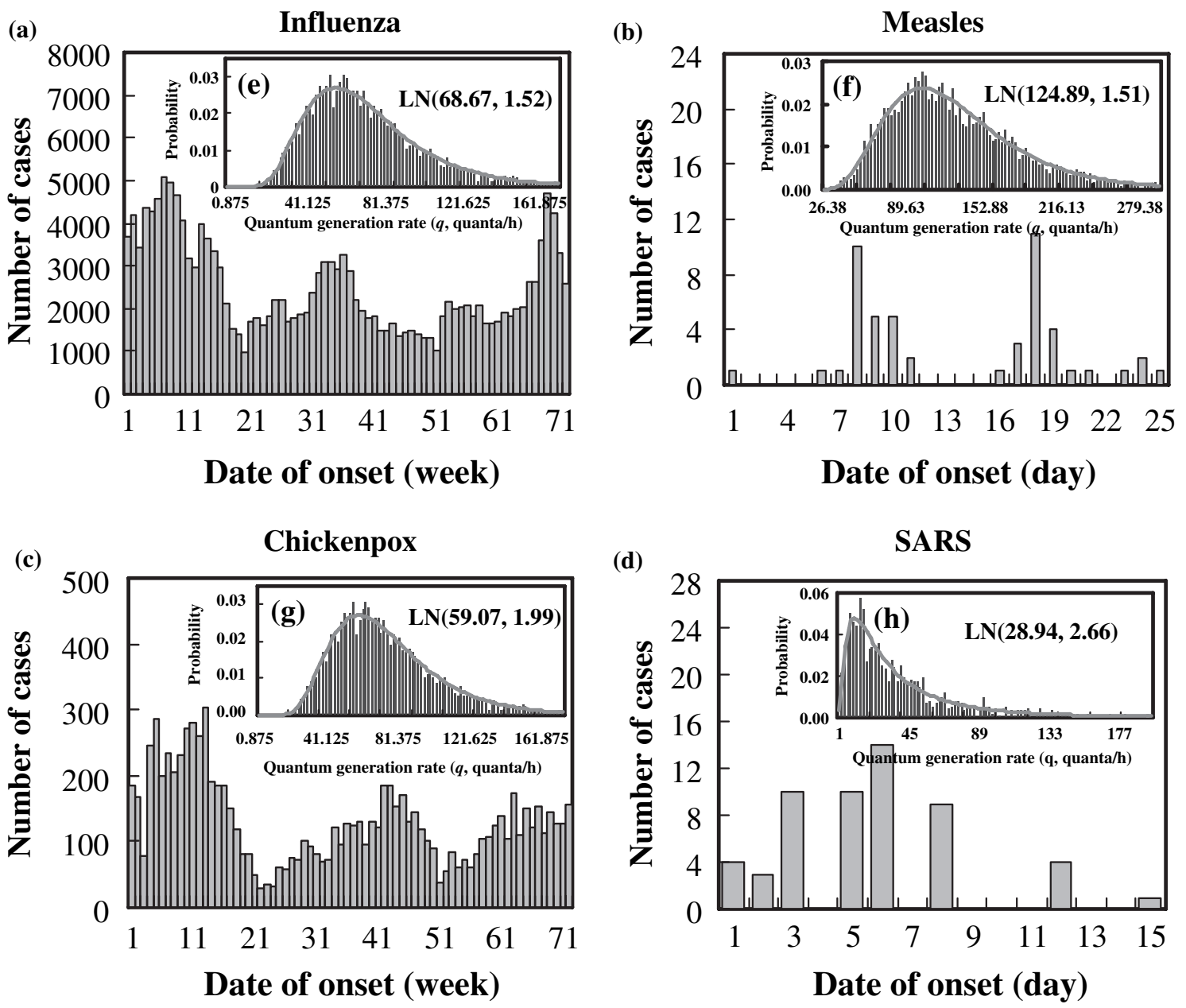

Fig. 1 Quantitative epidemiology of number of cases reported and the probability distribution of quantum generation rates calculated from the modified Wells-Riley equation (Equation 1) together with the estimated probability of infection $(P)$ and the adopted input parameters (Table 1) for influenza $(\mathrm{a}, \mathrm{e})$, measles $(\mathrm{b}, \mathrm{f})$, chickenpox $(\mathrm{c}, \mathrm{g})$, and SARS $(\mathrm{d}, \mathrm{h})$ 


\section{Chen et al.}

Table 1 Input parameters used in modified Wells-Riley mathematical equation to estimate the quantum generation rate $(q)$

\begin{tabular}{|c|c|c|c|c|c|c|c|}
\hline & $\begin{array}{l}\text { People in the } \\
\text { ventilated } \\
\text { airspace, } n\end{array}$ & $\begin{array}{l}\text { Volume of the } \\
\text { shared airspace, } \\
V\left(\mathrm{~m}^{3}\right)\end{array}$ & $\begin{array}{l}\text { Total } \\
\text { exposure } \\
\text { time, } t(\mathrm{~h})\end{array}$ & $\begin{array}{l}\text { Breathing } \\
\text { rate, }{ }^{a} p\left(m^{3} / h\right)\end{array}$ & $\begin{array}{l}\text { Fraction of indoor } \\
\text { air that is exhaled } \\
\text { breath, } f\end{array}$ & $\begin{array}{l}\text { Number of } \\
\text { infectors, I }\end{array}$ & $\begin{array}{l}\text { Probability of } \\
\text { infection, } P\end{array}$ \\
\hline \multicolumn{8}{|c|}{ Elementary school } \\
\hline Influenza & 50 & 600 & 6 & 0.38 & $0.00076^{\mathrm{b}}$ & 1 & $0.0052,1.51^{\mathrm{e}}$ \\
\hline \multicolumn{8}{|c|}{ Taipei Municipal Ho-Ping Hospital } \\
\hline SARS & 20,832 & 145,602 & 6 & 1.375 & $0.0247^{\mathrm{c}}$ & 1 & $0.00015,2.66$ \\
\hline \multicolumn{8}{|l|}{ Kindergarten } \\
\hline Chickenpox & 360 & 1656 & 5 & 0.32 & $0.00076^{\mathrm{b}}$ & 1 & $0.00045,2.71$ \\
\hline \multicolumn{8}{|c|}{ Elementary school $^{d}$} \\
\hline Measles & 616 & 4200 & 4 & 0.32 & 0.00464 & 1 & $0.0019,1.52$ \\
\hline
\end{tabular}

adopted from ICRP 66 (ICRP, 1994)

${ }^{\mathrm{b}}$ Calculated based on $Q=n p / f$ for $Q=20 \mathrm{~m}^{3} / \mathrm{h} / \mathrm{m}^{2}$ and floor area $=200 \mathrm{~m}^{2}$ (Construction and Planning Agency, Ministry of Interior, China).

${ }^{\mathrm{C}}$ Based on $\mathrm{Q}=5$ air changes per hour (ACH) (A standard regulation level of hospital emergency room by the Construction and Planning Agency, Ministry of Interior, China).

${ }^{\mathrm{d} A d o p t e d ~ f r o m ~ R i l e y ~ e t ~ a l . ~(1978) . ~}$

eLognormal distribution with a geometric mean and a geometric standard deviation.

Table 2 Input parameters of modified Wells-Riley mathematical model to estimate basic reproductive number $\left(R_{0}\right)$

\begin{tabular}{|c|c|c|c|c|c|c|c|c|}
\hline & $\mathrm{ACH}\left(\mathrm{h}^{-1}\right)$ & $\begin{array}{l}\text { People in the } \\
\text { ventilated } \\
\text { airspace, } n\end{array}$ & $\begin{array}{l}\text { Volume of } \\
\text { the shared } \\
\text { airspace, } V\left(\mathrm{~m}^{3}\right)\end{array}$ & $\begin{array}{l}\text { Total exposure } \\
\text { time, } t(\mathrm{~h})\end{array}$ & $\begin{array}{l}\text { Breathing } \\
\text { rate }^{\text {b }} \\
p\left(\mathrm{~m}^{3} / \mathrm{h}\right)\end{array}$ & $\begin{array}{l}\text { Fraction of indoor } \\
\text { air that is exhaled } \\
\text { breath, } f\end{array}$ & $\begin{array}{l}\text { Number of } \\
\text { infectors, I }\end{array}$ & $\begin{array}{l}\text { Estimated quantum } \\
\text { generation rate, } \\
q\left(\mathrm{~h}^{-1}\right)\end{array}$ \\
\hline \multicolumn{9}{|l|}{ Airplane $^{\mathrm{a}}$} \\
\hline Influenza & 5 & 54 & 168 & 6 & 0.48 & $0.0306^{c}$ & 1 & $68.67,1.52^{\mathrm{e}}$ \\
\hline \multicolumn{9}{|c|}{ Taiwan University Hospital } \\
\hline SARS & 5 & 300 & 7465.5 & 24 & 0.48 & $0.00386^{\mathrm{d}}$ & 1 & $28.94,2.66$ \\
\hline \multicolumn{9}{|l|}{ Airplane $^{a}$} \\
\hline Chickenpox & 5 & 54 & 168 & 6 & 0.48 & $0.0306^{c}$ & 1 & $59.07,1.99$ \\
\hline \multicolumn{9}{|l|}{ Airplane $^{a}$} \\
\hline Measles & 5 & 54 & 168 & 6 & 0.48 & $0.0306^{c}$ & 1 & $124.89,1.51$ \\
\hline
\end{tabular}

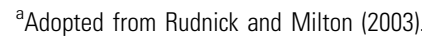

${ }^{\text {b} A d o p t e d ~ f r o m ~ I C R P ~} 66$ (ICRP, 1994).

${ }^{\mathrm{C}}$ Based on $\mathrm{Q}=0.3 \mathrm{ACH}$, a mean value adopted from Rudnick and Milton (2003).

${ }^{\mathrm{d} B a s e d}$ on $0=5 \mathrm{ACH}$ (Construction and Planning Agency, Ministry of Interior, China).

eLognormal distribution with a geometric mean and a geometric standard deviation.

After optimizing the KS statistics, lognormal distribution was the best fit for quantum generation rates of influenza, measles, chickenpox, and SARS (Figure 1eh). Figure $1 \mathrm{e}-\mathrm{h}$ indicates that measles has relatively largest quantum generation rate of $\mathrm{LN}(124.89$ quanta/h, 1.51) than that of influenza $[\mathrm{LN}(68.67,1.52)]$, chickenpox $[\operatorname{LN}(59.07,1.99)]$, and SARS $[\operatorname{LN}(28.94,2.66)]$.

Parameter estimates for basic reproductive number $\left(R_{0}\right)$ and proportion of asymptomatic infection $(\theta)$

We consider two highly infectious environmental settings, National Taiwan University (NTU) Hospital for SARS and aircraft Boeing 737 for influenza, chickenpox, and measles (Table 2), to assess the public health impact of control measures against indoor airborne infection. We used reported epidemic curves (Figure 1a-d) and proposed model in Equation 2 to quantify the uncertainty concerning basic reproductive number $\left(R_{0}\right)$ attributable to the large variance of infected probabilities and estimated quantum generation rates of selected airborne infection (Tables 1 and 2).
Table 2 gives the adopted assumption of the indoor environment such as the total exposure time and breathing rate in considering the normal conditions for four airborne infections, whereas we also assume the number of infectors is equal to 1 . Other parameters such as the people in ventilated airspace, volume of the shared airspace, and fraction of indoor air that is exhaled breath are adopted from three actual reported cases. Figure $2 \mathrm{a}-\mathrm{d}$ illustrates probability density functions of the optimized lognormal distributions of $R_{0}$ in that the Monte Carlo simulation results show $\mathrm{LN}(10.65,1.44), \mathrm{LN}(17.6,1.4), \mathrm{LN}(9.25,1.83)$, and $\mathrm{LN}(2.65,2.55)$ for influenza, measles, chickenpox, SARS, respectively. The most notable feature of the lognormal distributions of $R_{0}$ is the considerable right skew for chickenpox and SARS. Figure 2 also indicates that measles has the largest $R_{0}$ value $(\mathrm{gm}=17.61)$ than that of influenza (10.65), chickenpox (9.25), and SARS (2.65).

We could estimate the proportion of asymptomatic infection $(\theta)$ based on the definition of $\theta=$ (incubation period - latent period)/infectious period applied to 

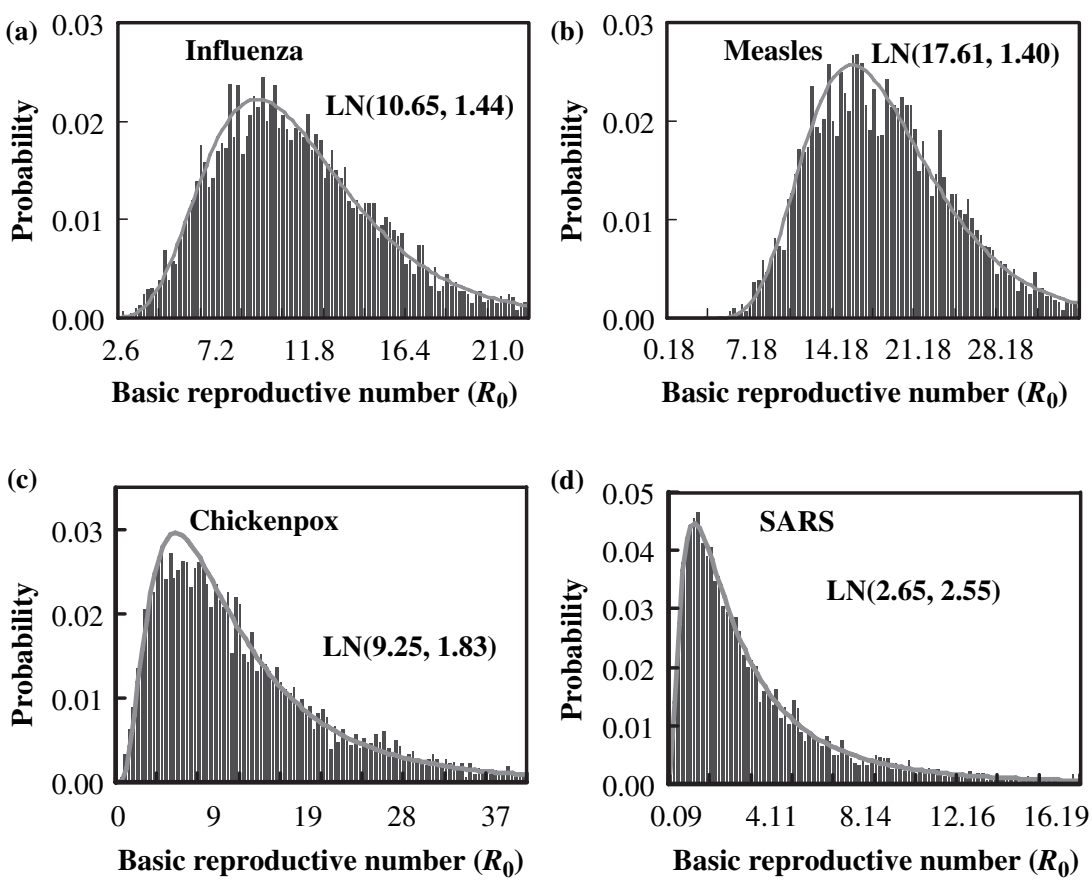

Fig. 2 Probability density functions of basic reproductive number $\left(R_{0}\right)$ followed the lognormal (LN) distributions of LN(10.65, 1.44), $\mathrm{LN}(17.6,1.4), \mathrm{LN}(9.25,1.83)$, and $\mathrm{LN}(2.65,2.55)$ for (a) influenza, (b) measles, (c) chickenpox, and (d) SARS, respectively

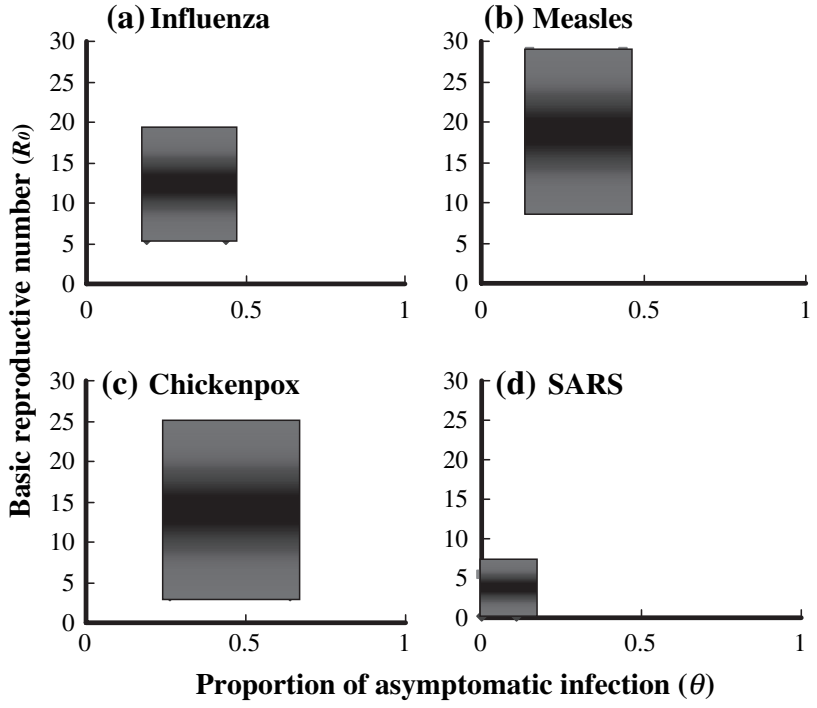

Fig. $3 R_{0}-\theta$ distributions with $90 \%$ confidence interval (CI) for four selected indoor airborne infections in that the $\theta$ and $R_{0}$ values, respectively, range from 0.19 to 0.44 and 5.53 to 18.49 for (a) influenza, 0.15 to 0.44 and 9.88 to 28.65 for (b) measles, 0.26 to 0.64 and 3.25 to 24.1 for (c) chickenpox, and 0.0001 to 0.11 and 0.25 to 5.31 for (d) SARS

four indoor airborne infections. We incorporate probability distribution into Monte Carlo simulation to obtain 5 th -95 th percentiles as $90 \%$ confidence interval (CI) for $R_{0}-\theta$ distributions (Figure $3 \mathrm{a}-\mathrm{d}$ ). The $90 \%$ CIs of $\theta$ range from $0.19-0.44,0.15-0.44,0.26-0.64$, and $0.0001-0.11$, whereas the $90 \%$ CIs of $R_{0}$ range from 5.53-18.49, 9.88-28.65, 3.25-24.1, and 0.25-5.31, for influenza, measles, chickenpox, and SARS, respectively. Figure 3 also reveals that SARS has the relatively smallest range of $\theta$ and $R_{0}(0.0001<\theta<0.11$ and $\left.0.25<R_{0}<5.31\right)$ than that of the other three indoor airborne infections.

Impact of engineering control measures

Following the concept of competing-risks model, we could reduce $R_{0}$ value from no control scenario by incorporating the engineering control measure strategies such as enhancing the efficiencies of recirculation air filter capacity and air change rate, as well as the respiratory protection by personal masking. We employed Equation 3 together with the adopted engineering control measures including enhancing air exchange rate and air filtration capacity as well as personal masking (Table 3) to estimate the controlled $R_{0}$, i.e. $R_{C}$, for selected four airborne infections. Apparently as indicated in Table $3, R_{C}$ values have efficiently reduced from no controlled $R_{0}$. For airborne infections such as influenza, chickenpox, and measles in an aircraft setting, engineering controls can provided a reliable control strategy to decrease the transmission potential and spread rate of an epidemic in that the efficacies range from $60 \%$ to $80 \%$ (Table 3 ). SARS, on the other hand, is predicted to be $60 \%$ efficacy with enhanced $\mathrm{ACH}$ and personal masking in a hospital setting (Table 3 ). 


\section{Chen et al.}

Table 3 Engineering control values of enhanced $\mathrm{ACH}$ and air filtration capacity, and personal masking efficiency of four airborne infections with the estimated controlled $R_{0}$ $\left(R_{C}\right)$

\begin{tabular}{llllll}
\hline & $\begin{array}{l}\text { Enhanced } \\
\mathrm{ACH} \\
\left(\mathrm{h}^{-1}\right)\end{array}$ & $\begin{array}{l}\text { Enhanced air } \\
\text { filtration } \\
\left(\mathrm{O}_{\mathrm{r}} \eta_{\mathrm{r}}\right)\left(\mathrm{m}^{3} / \mathrm{h}\right)\end{array}$ & $\begin{array}{l}\text { Masking } \\
\text { efficiency } \\
\left(\eta_{\mathrm{s}}\right)\end{array}$ & $R_{0}$ & $\begin{array}{l}\text { Derived } \\
R_{0}\left(R_{\mathrm{C}}\right)\end{array}$ \\
\hline $\begin{array}{l}\text { Airplane } \\
\quad \text { Influenza }\end{array}$ & $15^{\mathrm{a}}$ & $10.92^{\mathrm{c}}$ & 0 & $10.65,1.44^{\mathrm{e}}$ & $3.97,1.49$ \\
$\begin{array}{c}\text { Taiwan University Hospital } \\
\quad \text { SARS }\end{array}$ & $5^{\mathrm{b}}$ & 0 & $0.58^{\mathrm{d}}$ & $2.65,2.55$ & $1.13,2.57$ \\
$\begin{array}{c}\text { Airplane } \\
\text { Chickenpox }\end{array}$ & $15^{\mathrm{a}}$ & $10.92^{\mathrm{c}}$ & $0.58^{\mathrm{d}}$ & $9.25,1.83$ & $1.60,2.20$ \\
$\begin{array}{l}\text { Airplane } \\
\text { Measles }\end{array}$ & $15^{\mathrm{a}}$ & $10.92^{\mathrm{c}}$ & $0.58^{\mathrm{d}}$ & $17.61,1.40$ & $3.06,1.50$ \\
\hline
\end{tabular}

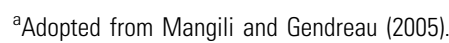

${ }^{\mathrm{b}}$ Adopted from ICRP 66 (ICRP, 1994).

${ }^{\mathrm{C}}$ Adopted from Lee et al. (2005).

${ }^{\text {d} A d o p t e d ~ f r o m ~ N a z a r o f f ~ e t ~ a l . ~(1998) . ~}$

'Lognormal distribution with a geometric mean and a geometric standard deviation.

Impact of public health interventions

Here we investigated intervention strategies chosen to demonstrate the impact of four selected airborne infections, as characterized by $R_{0}-\theta$ curve, on the efficacy of public health intervention. We considered $100 \%$ effective isolation of symptomatic patients (i.e., $\varepsilon_{1}=1$ ) and ranged values of effective vaccination (i.e., $\varepsilon_{2}$ ) adopted from published empirical data (Table 4) for influenza, chickenpox, and measles. For SARS, we
Table 4 Public health interventions of effective isolation, vaccination, and contact tracing of four airborne infections with the estimated uncontrollable ratios

\begin{tabular}{|c|c|c|c|}
\hline $\begin{array}{l}\text { Airborne } \\
\text { infection }\end{array}$ & $\begin{array}{l}\text { Effectiveness } \\
\# 1\left(\varepsilon_{1}\right)(\%)\end{array}$ & $\begin{array}{l}\text { Effectiveness } \\
\# 2\left(\varepsilon_{2}\right)(\%)\end{array}$ & $\begin{array}{l}\text { Uncontrollable } \\
\text { ratio }(\%)\end{array}$ \\
\hline Influenza & Isolation (100) & Vaccination $\left(70-90^{\mathrm{a}}\right)$ & $93.5-97.9$ \\
\hline SARS & Isolation (100) & Contact tracing [50 (30-80)] & $0-1.2$ \\
\hline Chickenpox & Isolation (100) & Vaccination $\left(73-95^{b}\right)$ & $96.7-98.7$ \\
\hline Measles & Isolation (100) & Vaccination $\left[73(32-89)^{c}\right]$ & $99.7-100$ \\
\hline
\end{tabular}

${ }^{a}$ Adopted from Couch (1999).

${ }^{\mathrm{b}}$ Adopted from Vazquez et al. (2005).

${ }^{c}$ Mean (min, max) that is adopted from De Serres et al. (1996).

considered $100 \%$ effective isolation along with the ranged value of effective contact tracing (Table 4). Criteria for outbreak control of $R_{0}-\theta$ critical control line that separates epidemic growth (above the line) from outbreak control (below the line) can be determined by Equation 4 with two assigned public health control measures (Figure 4).

Each control line represents a specific scenario, consisting of various control efficacies in that the dark color lines denote the higher efficacy, whereas the light color lines indicate the lower efficacy of the chosen control measures (Figure $4 \mathrm{a}-\mathrm{d}$ ). For each scenario, if a given airborne infection is below the $R_{0}-\theta$ control line, the outbreak is always controlled eventually. Areas above the control line indicate that additional control measures would be required to control spread

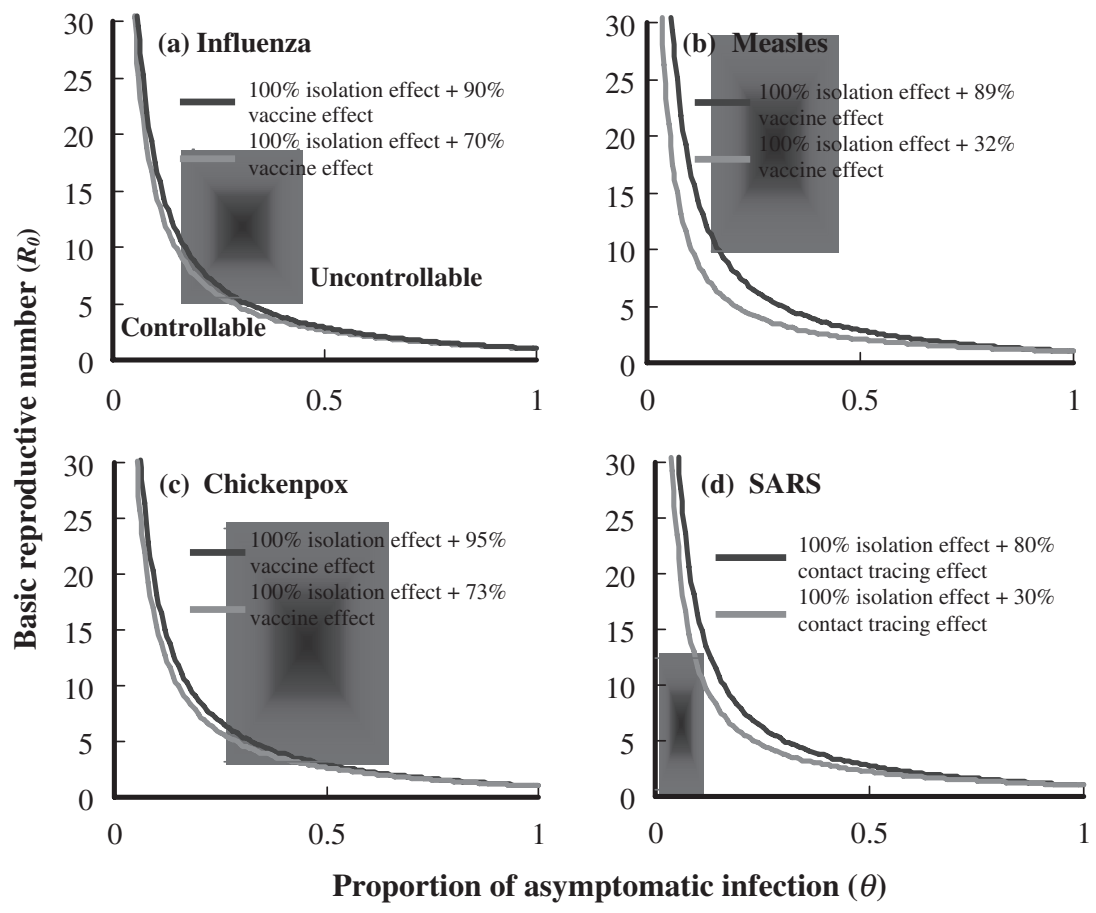

Fig. 4 Criteria for outbreak control of $R_{0}-\theta$ critical control line that separates epidemic growth (above the line) from outbreak control (below the line). We examine the effectiveness of two public health control measures including isolation and vaccine for (a) influenza, (b) measles, and (c) chickenpox, whereas isolation and contact tracing for (d) SARS in containing the spread of the disease at the early-stage of outbreak 
rate. We calculated the percentage of uncontrollable ratio which varied with the control efficacies, demonstrating $93.5-97.9 \%, 0-1.2 \%, 96.7-98.7 \%$, and $99.7-$ $100 \%$ for influenza, SARS, chickenpox, and measles, respectively (Table 4 ).

Comparing $R_{0}-\theta$ control lines for influenza, measles, chickenpox, and SARS (Figure 4 and Table 4), it is clear that SARS is the easiest of the four airborne infections to control because of its low $R_{0}$ and $\theta$ values. Our analysis indicates that effective isolation of symptomatic patients with low-efficacy contact tracing is sufficient to control a SARS outbreak (Figure 4d). On the other hand, influenza, measles, and chickenpox are predicted to be very difficult to control even with $100 \%$ effective isolation along with $90-95 \%$ effective vaccination. Therefore, additional control measures have to be incorporated into public health intervention strategies.
Combinations of engineering controls and public health interventions

We further investigated the efficacy of some combinations of engineering control measures with public health interventions in containing epidemic growth of influenza, chickenpox, and measles. The chosen combinations of the engineering control measures and public health interventions of four selected airborne infections given in Table 5 are used to predict the uncontrollable ratio in $R_{0}-\theta$ curves. Figure $5 \mathrm{a}-\mathrm{d}$ represents the criteria for outbreak control lines by combination with public health interventions and engineering control measures for influence, measles, chickenpox, and SARS, respectively.

For influenza, if we apply $100 \%$ effective isolation with two different vaccine effects of $70 \%$ and $90 \%$ (Figure 5a) combined with three engineering control

Table 5 Combination of the engineering control measure and public health intervention control measures of four airborne infections to predict the uncontrollable ratio

\begin{tabular}{|c|c|c|c|c|c|c|}
\hline $\begin{array}{l}\text { Airborne } \\
\text { infection }\end{array}$ & $\begin{array}{l}\text { Enhanced } \\
\mathrm{ACH}\left(\mathrm{h}^{-1}\right)\end{array}$ & $\begin{array}{l}\text { Enhanced air } \\
\text { filtration }\left(Q_{r} \eta_{r}\right) \\
\left(\mathrm{m}^{3} / \mathrm{h}\right)\end{array}$ & $\begin{array}{l}\text { Masking } \\
\text { efficiency }\left(\eta_{\mathrm{s}}\right)\end{array}$ & $\begin{array}{l}\text { Effectiveness } \\
\# 1\left(\varepsilon_{1}\right)(\%)\end{array}$ & $\begin{array}{l}\text { Effectiveness } \\
\# 2\left(\varepsilon_{2}\right)(\%)\end{array}$ & $\begin{array}{l}\text { Uncontrollable } \\
\text { ratio }(\%)\end{array}$ \\
\hline Influenza & 15 & 10.92 & 0 & Isolation (100) & Vaccination $\left(70-90^{\mathrm{a}}\right)$ & $39.6-50.9$ \\
\hline SARS & 5 & 0 & 0.58 & Isolation (100) & Contact tracing [50 (30-80)] & $0-0$ \\
\hline Chickenpox & 15 & 10.92 & 0.58 & Isolation (100) & Vaccination $\left(73-95^{\mathrm{b}}\right)$ & $40.8-48.3$ \\
\hline Measles & 15 & 10.92 & 0.58 & Isolation (100) & Vaccination [73 (32-89) $\left.{ }^{\mathrm{C}}\right]$ & $20.2-37.7$ \\
\hline
\end{tabular}

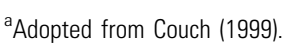

${ }^{\mathrm{b}}$ Adopted from Vazquez et al. (2005).

${ }^{c}$ Mean (min, $\max$ ) that is adopted from De Serres et al. (1996).

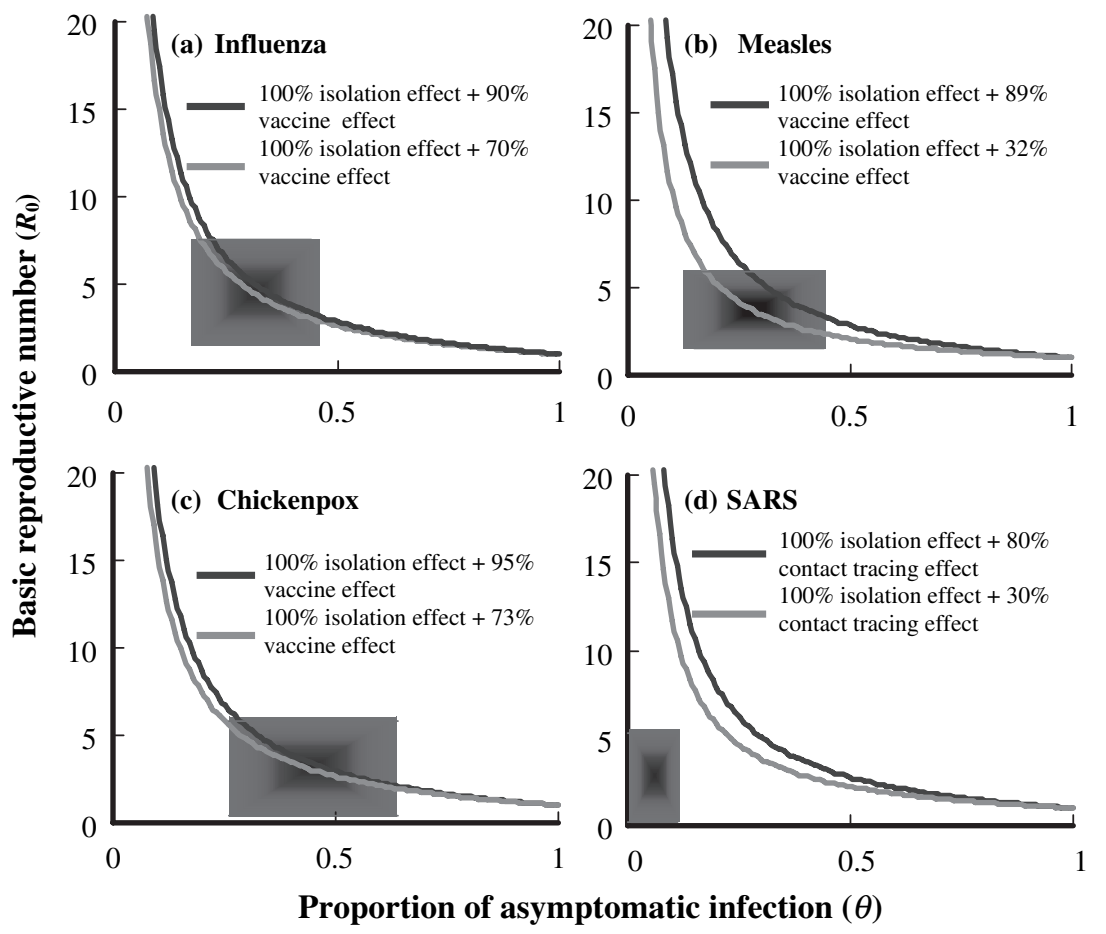

Fig. 5 Criteria for outbreak control lines by combination of public health interventions and engineering control measures for (a) influenza, (b) measles, (c) chickenpox, and (d) SARS, respectively 


\section{Chen et al.}

measures including enhanced $\mathrm{ACH}$ and air filtration capacity as well as personal masking, resulting predicted uncontrollable ratios range from $40 \%$ to $51 \%$ that are much lower than that of $94-98 \%$ with no engineering control measures involved. For measles and chickenpox, on the other hand, the $100 \%$ effective isolation and two different vaccine effects of $32 \%$ and $73 \%$ with additional engineering control measures (Table 5), the uncontrollable ratios are dramatically decrease from $97 \%$ to $100 \%$ with no engineering control implemented to $20-38 \%$ and $41-48 \%$, respectively (Figure 5b,c).

\section{Discussion}

Based on our modeling methodology and results, four crucial points are discussed: (i) Why influenza and chickenpox still have as high as $50 \%$ uncontrollable ratio? (ii) How will we know which one is the most suitable control strategy for a specific indoor airborne infection? (iii) Should additional effective interventions be implemented, how to manipulate the criteria for outbreak control $R_{0}-\theta$ critical curve to contain the airborne infection or a newly emergent infectious disease? (iv) Future challenges toward the control and transmission of airborne infections.

Why do influenza and chickenpox still have uncontrollable ratio as high as $50 \%$ ? The epidemiological data and the biology of the etiological agent may provide the explanations. First, the empirical data of distribution of chickenpox and influenza cases adopted are from January 2003 to April 2005 and to date there is still no free full-scale vaccination in Taiwan, with the result that the vaccination coverage rate is too low to control airborne infections, although the vaccination effectiveness of chickenpox and influenza range from $70-95 \%$ (Table 5). The specific vaccination policy may depend on the target population of interest, ethnic groups, geographic areas, epidemic strain of virus, disease seasonality, and consultations and admission rates varying from country to country (Wang et al., 2005). Secondly, there are higher levels of asymptomatic transmission $(\theta)$ for influenza (Fraser et al., 2004) and chickenpox. Even if we carry out the efficacy of public health interventions in the presence of engineering control measures, the probability of success for containing influenza and chickenpox is still not significant.

Lee et al. (2005) proposed three intervention categories including personal measures, administrative controls, and engineering controls for containing infectious acute respiratory disease. Engineering controls are generally considered more reliable than other intervention categories as they do not require individual compliance or enforcement of administrative policies (Lee et al., 2005). Vaccination remains the primary public health intervention and has been demonstrated to reduce influenza-related morbidity. However, the antigenic variability of influenza virus necessitates continuous surveillance to identify new vaccine candidate strains, and vaccine efficacy is reduced in key target groups such as the elderly and immunocompromised (Ferguson et al., 2003). However, this modeling approach of Wells-Riley mathematical equation has two assumptions: (i) a well-mixed airspace, and (ii) steady-state conditions. These assumptions simplify ventilation condition when modeling the control strategies, and thus we did not consider the interaction between the infiltration flow and ventilation flow.

Our results also imply that control measures based on case identification such as contact tracing, isolation, vaccination and treatment, that might be central to the control of one specific airborne infection, will only be partially successfully for the other infectious disease. For example, for influenza, unlike SARS, substantial transmission occurs before the onset of case-defining symptoms. This implies that measures that generally reduce contacts between persons, regardless of infection status, may be our most powerful protection against a pandemic until adequate vaccine and antiviral medicines can be produced, at which point mass-vaccination and prophylaxis may be more effective than targeted approaches (Mills et al., 2004).

Here we show that the approach presented is scalable and can be extended to include additional control efficacies. Taking influenza as an example, we incorporate one additional public health intervention of handwashing to simulate the controllable level in the presence of three effective public health interventions and enhanced engineering control measures. The $R_{0}-\theta$ critical control line can be determined by the following equation:

$$
\begin{aligned}
R_{0} & \left\{\left(1-\varepsilon_{1}\right)\left(1-\varepsilon_{2}\right)\left(1-\varepsilon_{3}\right)+\varepsilon_{1}\left(1-\varepsilon_{2}\right)\left(1-\varepsilon_{3}\right) \theta\right. \\
& +\varepsilon_{2}\left(1-\varepsilon_{1}\right)\left(1-\varepsilon_{3}\right) \theta+\varepsilon_{3}\left(1-\varepsilon_{1}\right)\left(1-\varepsilon_{2}\right) \theta \\
& +\left[\varepsilon_{1} \varepsilon_{2}\left(1-\varepsilon_{3}\right)+\varepsilon_{1} \varepsilon_{3}\left(1-\varepsilon_{2}\right)+\varepsilon_{2} \varepsilon_{3}\left(1-\varepsilon_{1}\right)\right] \\
& {\left.[\theta /(2-\theta)]+\varepsilon_{1} \varepsilon_{2} \varepsilon_{3}[\theta /(3-2 \theta)]\right\}=1, }
\end{aligned}
$$

where $\varepsilon_{3}$ is the effectiveness of handwashing was $45 \%$ (Ryan et al., 2001). The estimated uncontrollable ratios range from $28 \%$ to $31 \%$ compared with $40-51 \%$ with no hand washing involved. The result indicates that the percentage reduction in incidence of influenza would be relatively modest. Despite incorporating additional public health interventions, we still could not fully control the pandemic of influenza. Our results are probabilistic and demonstrate considerable variability in the potential size of the epidemic in the absence of and in response to engineering control and public health intervention. Public health officials need to keep this probabilistic characteristic of success in mind when planning and evaluating their response. We have 
developed a flexible mathematical model that can help determine the best intervention strategies for containing indoor airborne infections at the early stage of outbreak.

However, beginning in the early 1990s, a new wave of epidemiological research emphasizes the transmission of heterogeneity (Ferguson et al., 2003). Modeling the contact probability among different age-specific force of infection even at the social networks framework (Eubank et al., 2004) will be a key point to describe the transmission patterns realistically. For the cluster group of human contact network in the indoor settings, person-to-person transmission will be a quick way to spread airborne infections. Therefore, it is important to model the infectious disease outbreaks varying with the age and social-specific force of infection and to describe the higher risk subpopulation or high transmission settings by control strategies. Further research will pinpoint the contact network epidemiology to explicitly capture patterns of disease transmission and thus enable more accurate and detailed predictions of the effect of control measures on the magnitude and distributions of an outbreak of indoor airborne infection.

\section{Acknowledgements}

We thank members of our lab, Dr Huang-Min Liang and $\mathrm{Mr} \mathrm{Li}-\mathrm{J}$ ohn Jou, for discussions and comments on manuscript.

\section{References}

Anderson, R.M. and May, R.M. (1991) Infectious Diseases of Humans: Dynamics and Control, Oxford, Science Publications.

Anderson, R.M., Fraser, C., Ghani, A.C., Donnelly, C.A., Riley, S., Ferguson, N.M., Leung, G.M., Lam, T.H. and Hedley, A.J. (2004) Epidemiology, transmission dynamics and control of SARS: the 2002-2003 epidemic, Phil. Trans. R. Soc. Lond. B, 359, 1091-1105.

Barnhart, S., Sheppard, L., Beaudet, N., Stover, B. and Balmes, J. (1997) Tuberculosis in health care settings and the estimated benefits of engineering controls and respiratory protection, J. Occup. Environ. Med., 39, 849-854.

Beggs, C.B. (2003) The airborne transmission of infection in hospital buildings: fact or fiction? Indoor Built. Environ., 12, 9-18.

Brookmeyer, R., Johnson, E. and Bollinger, R. (2003) Modeling the optimum duration of antibiotic prophylaxis in an anthrax outbreak, Proc. Natl. Acad. Sci. U.S.A., 100, 10129-10132.

Brookmeyer, R., Johnson, E. and Bollinger, R. (2004) Public health vaccination policies for containing an anthrax outbreak, Nature, 432, 901-904.

Brookmeyer, R., Johnson, E. and Barry, S. (2005) Modeling the incubation period of anthrax, Stat. Med., 24, 531-542.

Chow, T.T. and Yang, X.Y. (2004) Ventilation performance in operating theatres against airborne infection: review of research activities and practical guidance, J. Hosp. Infect., 56, 85-92.

Couch, R.B. (1999) Measures for control of influenza, Pharmacoeconomics, 16 (Suppl.), 41-45.

De Serres, G., Boulianne, N., Ratnam, S. and Corriveau, A. (1996) Effectiveness of vaccination at 6 to 11 months of age during an outbreak of measles, Pediatrics, 97, 232-235.
Donnelly, C.A., Ghani, A.C., Leung, G.M., Hedley, A.J., Fraser, C., Riley, S., Abu-Raddad, L.J., Ho, L.M., Thach, T.Q., Chau, P., Chan, K.P., Lam, T.H., Tse, L.Y., Tsang, T., Liu, S.H., Kong, J.H.B., Lau, E.M.C., Ferguson, N.M. and Anderson, R.M. (2003) Epidemiological determinants of spread of causal agent of severe acute respiratory syndrome in Hong Kong, Lancet, 361, 17611766.

Eubank, S., Guclu, H., Anil Kumar, V.S., Marathe, M.V., Srinivasan, A., Toroczkai, Z. and Wang, N. (2004) Modeling disease outbreaks in realistic urban social networks, Nature, 429, 180-184.

Fauci, A.S., Touchette, N.A. and Folkers, G.K. (2005) Emerging infectious diseases: a 10-year perspective from the National Institute of Allergy and Infectious Diseases, Emerg. Infect. Dis., 11, 519-525.

Fennelly, K.P., Davidow, A.L., Miller, S.L., Connell, N. and Ellner, J.J. (2004) Airborne infection with Bacillus anthracis from mills to mail, Emerg. Infect. Dis., 10, 996-1001.

Ferguson, N.M., Mallett, S., Jackson, H., Roberts, N. and Ward, P. (2003) A population-dynamic model for evaluating the potential spread of drug-resistant influenza virus infections during communitybased use of antivirals, J. Antimicrob. Chemother., 51, 977-990.

Fraser, C., Riley, S., Anderson, R.M. and Ferguson, N.M. (2004) Factors that make an infectious disease outbreak controllable, Proc. Natl. Acad. Sci. U.S.A., 101, 6146-6151.

ICRP (1994) Human Respiratory Tract Model for Radiological Protection, a Report of Task Group of the International Commission on Radiological Protection, ICRP Publication, Vol. 66, New York, Elsevier.

Lee, S.A., Grinshpun, S.A., Adhikari, A., Li, W.X., McKay, R., Maynard, A. and
Reponen, T. (2005) Laboratory and field evaluation of a new personal sampling system for assessing the protection provided by the N95 filtering face piece respirators against particles, Ann. Occup. Hyg., 49, 245-257.

Li, Y., Duan, S., Yu, I.T.S. and Wong, T.W. (2005a) Multi-zone modeling of probable SARS virus transmission by airflow between flats in Block E, Amoy Gardens, Indoor Air, 15, 96-111.

Li, Y., Huang, X., Yu, I.T.S., Wong, T.W. and Qian, H. (2005b) Role of air distribution in SARS transmission during the largest nosocomial outbreak in Hong Kong, Indoor Air, 15, 83-95.

Mangili, A. and Gendreau, M.A. (2005) Transmission of infectious diseases during commercial air travel, Lancet, 365, 989996.

Mills, C.E., Robins, J.M. and Lipsitch, M. (2004) Transmissibility of 1918 pandemic influenza, Nature, 432, 904-906.

Morens, D.M., Folkers, G.K. and Fauci, A.S. (2004) The challenge of emerging and re-emerging infectious diseases, Nature, 430, 242-249.

Murray, J.D. (2001) Mathematical Biology I: an Introduction, New York, Springer Publishing Co.

Myatt, T.A., Johnston, S.L., Zuo, Z.F., Wand, M., Kebadze, T., Rudnick, S. and Milton, D.K. (2004) Detection of airborne rhinovirus and its relation to outdoor air supply in office environments, Am. J. Resp. Crit. Care., 169, 1187-1190.

Nardell, E.A., Keegan, J., Cheney, S.A. and Etkind, S.C. (1991) Airborne infection: theoretical limits of protection achievable by building ventilation, Am. Rev. Respir. Dis., 144, 302-306.

Nazaroff, W.W., Nicas, M. and Miller, S.L. (1998) Framework for evaluating measures to control nosocomial tuberculosis transmission, Indoor Air, 8, 205-218. 


\section{Chen et al.}

Riley, E.C., Murphy, G. and Riley, R.L. (1978) Airborne spread of measles in a suburban elementary-school, Am. J. Epidemiol., 107, 421-432.

Rudnick, S.N. and Milton, D.K. (2003) Risk of indoor airborne infection transmission estimated from carbon dioxide concentration, Indoor Air, 13, 237-245.

Ryan, M.A.K., Christian, R.S. and Wohlrabe, J. (2001) Handwashing and respiratory illness among young adults in military training, Am. J. Prev. Med., 21, 79-83.

Vazquez, M., LaRussa, P.S., Gershon, A.A., Niccolai, L.M., Muehlenbein, C.E., Steinberg, S.P. and Shapiro, E.D. (2005) Effectiveness over time of varicella vaccine, J. Am. Med. Assoc., 291, 851-855.
Wang, S.T., Lee, L.T., Chen, L.S. and Chen, T.H.H. (2005) Economic evaluation of vaccination against influenza in the elderly: an experience from a populationbased influenza vaccination program in Taiwan, Vaccine, 23, 1973-1980.

WHO (2003a) Cumulative Number of Reported Probable Cases of SARS. From 1 November 2002 to 11 July 2003. (http:// www.who.int/csr/sars/country/ 2003_07_11/en).

WHO (2003) Influenza Overview (http:// www.who.int/topics/influenza/en).

WHO (2003c) SARS Situation in Taiwan. (http://www.who.int/csr/don/ 2003_05_28/en).
WHO (2004) The World Health Report 2004 - Changing History (http://www.who.int/ whr/2004/en).

Wong, T.W., Lee, C.K., Tam, W., Lau, J.T.F., Yu, T.S., Lui, S.F., Chan, P.K.S., Li, Y., Bresee, J.S., Sung, J.J.Y. and Parashar, U.D. (2004) Cluster of SARS among medical students exposed to single patient, Hong Kong, Emerg. Infect. Dis., 10, 269-276.

Yu, I.T., Li, Y., Wong, T.W., Tam, W., Chan, A.T., Lee, J.H., Leung, D.Y. and Ho, T. (2004) Evidence of airborne transmission of the severe acute respiratory syndrome virus, N. Engl. J. Med., 22, 1731-1739.

\section{Appendix}

Derivation of quantum generation rate $(q)$

The infection probability can be given as,

$$
P=\frac{D}{S},
$$

where $S$ is the number of susceptible and $D$ is the number of cases for four airborne infections. The input susceptible numbers are 368,404, 368,404, 616, and 20,832 for influenza, chickenpox, measles, and SARS, respectively. The quantum generation rate $q$ can then be derived directly from Equation 1 as:

$$
q=\frac{-n \ln (1-P)}{I f t\left\{1-\frac{V f}{n p t}\left[1-\exp \left(-\frac{n p t}{V f}\right)\right]\right\}} .
$$

\section{Combining competing-risks model with Wells-Riley equation}

Based on the competing-risks model, the generation probability of infectious agent within $t$ hours will be

$$
\int_{0}^{t} \lambda \mathrm{e}^{-\lambda \tau} \mathrm{e}^{-C \tau} \mathrm{d} \tau=\frac{\lambda}{\lambda+C}\{1-\exp [-(\lambda+C) t]\},
$$

where $C$ is the cleared rate combined with $\mathrm{ACH}$ (i.e., $C=\mathrm{ACH}+\mathrm{ACH}_{\mathrm{r}} \eta_{\mathrm{r}}$ ) to decrease the risk of infectious agents, and $\lambda$ is the generation rate to increase the risk of infectious agents $\left[\lambda=p\left(1-\eta_{\mathrm{s}}\right) / V\right]$, and the infectious agent dose $D$ is: $D=I q$.

Thus, the probability that at least one of $D$ infectious agent generation with a Poisson distribution will be:

$$
P=1-\exp \left[-D \frac{\lambda}{\lambda+C}[1-\exp (-(\lambda+C) t)]\right] .
$$

We simplify Equation 10 for $\lambda<<C$,

$$
P=1-\exp \left[-D \frac{\lambda}{C}(1-\exp (-C t))\right]
$$

Equation 3 can be rewritten by incorporating the definitions of $C, \lambda$, and $D$ associated with the definitions of $Q=V \times \mathrm{ACH}$ and $Q_{\mathrm{r}}=V \times \mathrm{ACH}_{\mathrm{r}}$ into Equation 11 as:

$$
\begin{aligned}
& P=1-\exp \left\{-\left[\frac{\operatorname{Iqtp}\left(1-\eta_{\mathrm{s}}\right)}{Q+Q_{\mathrm{r}} \eta_{\mathrm{r}}}\right]\right. \\
& \left.\left[1-\exp \left(-\left(A C H+A C H_{\mathrm{r}} \eta_{\mathrm{r}}\right) t\right)\right]\right\} .
\end{aligned}
$$

Therefore Equation 3 could be derived by incorporating Equation 12 into Equation 7 as $R_{0}=S \times P=$ $(n-1) \times P$.

Combining $R_{0}-\theta$ critical control line with Von Foerster equation

We deliberately manipulate the mathematical derivations to arrive at the critical control line originally appeared in Fraser et al. (2004). Based on the following algebraic equation

$$
\int_{0}^{\infty} \beta(\tau)\left[1-\varepsilon_{1}+\varepsilon_{1} S(\tau)\right]\left[1-\varepsilon_{2}+\varepsilon_{2} S(\tau)\right] \mathrm{d} \tau=1,
$$

we incorporate the definitions of $\beta(\tau)=R_{0} \mathrm{e}^{-\tau}, S(\tau)=$ $\mathrm{e}^{-v \tau}, \theta=1 /(v+1)$ into Equation 3, obtaining 


$$
\begin{aligned}
& \int_{0}^{\infty} R_{0}\left[\mathrm{e}^{-\tau}-\varepsilon_{1} \mathrm{e}^{-\tau}-\varepsilon_{2} \mathrm{e}^{-\tau}+\varepsilon_{1} \varepsilon_{2} \mathrm{e}^{-\tau}+\varepsilon_{1} \mathrm{e}^{-(v+1) \tau}\right. \\
& \left.+\varepsilon_{2} \mathrm{e}^{-(v+1) \tau}-2 \varepsilon_{1} \varepsilon_{2} \mathrm{e}^{-(v+1) \tau}+\varepsilon_{1} \varepsilon_{2} \mathrm{e}^{-(2 v+1) \tau}\right] \mathrm{d} \tau \\
& =R_{0}\left[\frac{\mathrm{e}^{-\tau}}{-\tau}\left(1-\varepsilon_{1}\right)\left(1-\varepsilon_{2}\right)-\frac{\mathrm{e}^{-\tau}}{v+1} \varepsilon_{1}\left(1-\varepsilon_{2}\right)\right. \\
& \left.\quad-\frac{\mathrm{e}^{-\tau}}{v+1} \varepsilon_{2}\left(1-\varepsilon_{1}\right)+\varepsilon_{1} \varepsilon_{2} \frac{-1}{2 v+1} \mathrm{e}^{-(2 v+1) \tau}\right]_{0}^{\infty}=1
\end{aligned} .
$$$$
\frac{1}{2 v+1}=\frac{\theta}{2-\theta} \text {. }
$$

We finally incorporate Equation 15 into Equation 14 to arrive at Equation 4 as:

$$
\begin{aligned}
& R_{0}\left[\left(1-\varepsilon_{1}\right)\left(1-\varepsilon_{2}\right)+\varepsilon_{1}\left(1-\varepsilon_{2}\right) \theta+\left(1-\varepsilon_{1}\right) \varepsilon_{2} \theta\right. \\
& \left.\quad+\varepsilon_{1} \varepsilon_{2} \frac{\theta}{2-\theta}\right]=1
\end{aligned}
$$

We rearrange the definitions between $v$ and $\theta$ as: $v=$ $(1 / \theta)-1$ to show that 
Copyright of Indoor Air is the property of Blackwell Publishing Limited and its content may not be copied or emailed to multiple sites or posted to a listserv without the copyright holder's express written permission. However, users may print, download, or email articles for individual use. 